R. M. BUCKLE AND W. D. NICHOL: DYSPHAGIA DUE TO MONILIAL OESOPHAGITIS
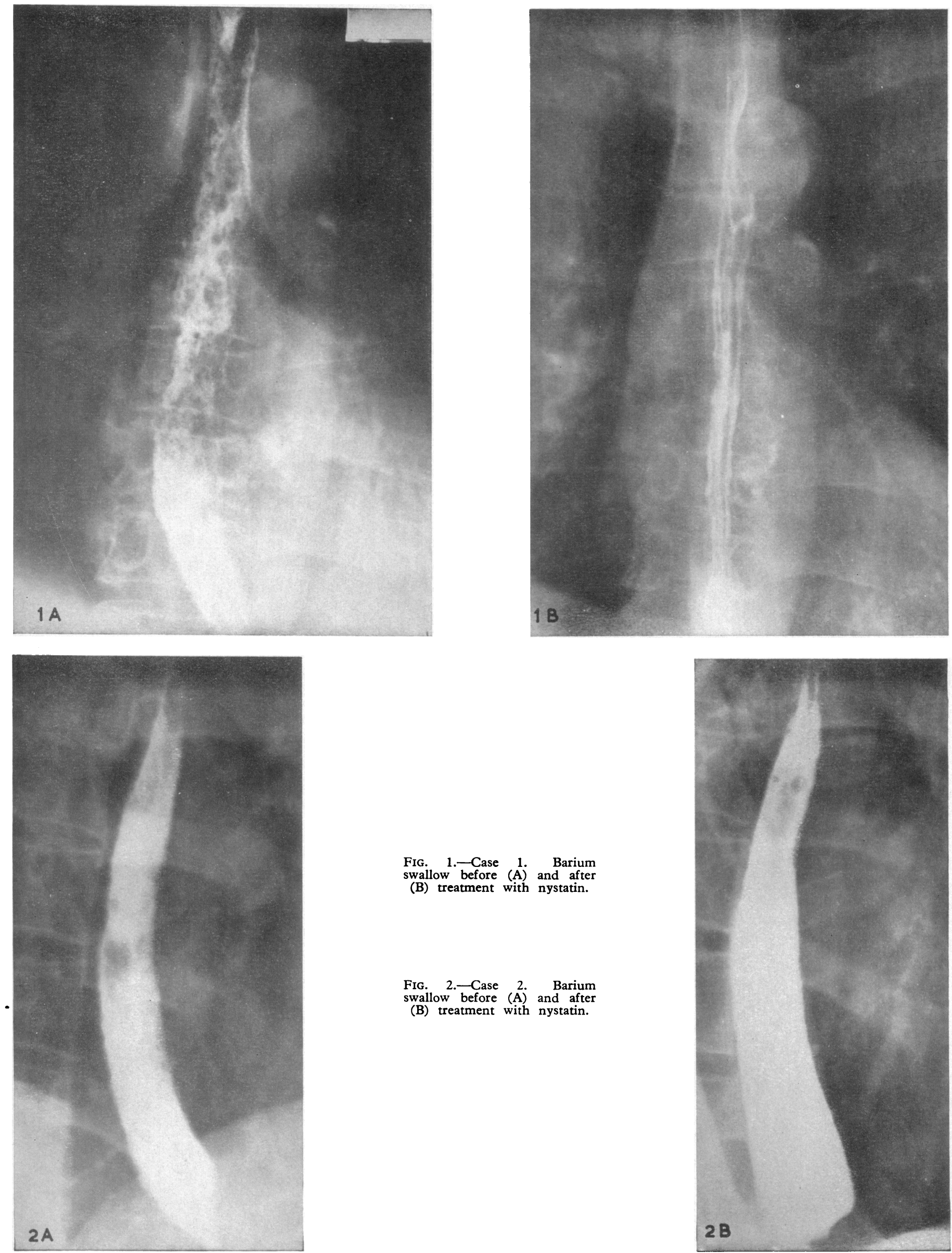


\title{
Painful Dysphagia Due to Monilial Oesophagitis
}

\author{
RICHARD M. BUCKLE,* M.A., M.D., M.R.C.P.; W. D. NICHOL,† M.B., CH.B., F.F.R., A.D.M.R.
}

\author{
[With Special Plate]
}

Brit. med. F., 1964, 1, 821-822

Spread of oral thrush to the oesophagus is uncommon in adults, but may occur as an incidental event during the terminal stages of infection in moribund patients. However, on occasion oesophageal involvement may be the dominant feature of a monilial infection. This report describes two patients who presented with retrosternal dysphagia due to monilial oesophagitis but in whom there was little evidence of thrush elsewhere, and illustrates the value of radiology in assisting in diagnosis.

\section{Case 1}

A 54-year-old man was seen with acute leukaemia. Apart from pallor and hepatomegaly, physical examination was normal. He was transfused and treated with prednisolone, $15 \mathrm{mg} . /$ day, and mercaptopurine, $150 \mathrm{mg}$./day. One week later he developed retrosternal pain on swallowing, but otherwise there was no deterioration in his condition and no elevation of the temperature or pulse. Treatment with antacids was without effect and the dysphagia worsened. He then developed basal pneumonia, for which tetracycline was given. During the next few days the dysphagia became so severe that only fluids could be swallowed. A barium swallow (Special Plate, Fig. 1 A) showed that the entire length of the thoracic oesophagus had a shaggy, irregular outline, and multiple small circular filling defects were seen along its length; these appearances suggested monilial oesophagitis. Examination of the mouth and oropharynx, however, showed no evidence of thrush ; but secretions from the hypopharynx, obtained by laryngeal swabbing, and swallowed oseophageal secretions, obtained by gastric aspiration, showed many yeast spores and pseudomycelia, together with desquamated epithelium invaded by pseudomycelia, and subsequent culture grew Candida albicans.

Tetracycline was discontinued, prednisolone was reduced to $10 \mathrm{mg} . /$ day, and nystatin was given, as a mucilage, 500,000 units every six hours. A rapid improvement occurred; swallowing eased within 36 hours and dysphagia completely disappeared by the fourth day. Nystatin was then reduced to 250,000 units six-hourly and finally stopped after 10 days. The pharyngeal secretions and stools no longer contained any yeasts, and a repeat barium swallow nine days after starting treatment showed a normal oesophagus (Special Plate, Fig. 1 B).

\section{Case 2}

A 30-year-old woman was admitted to hospital with acute ulcerative colitis. Treatment with sulphasalazine, $1 \mathrm{~g}$. six-hourly, and prednisolone, $5 \mathrm{mg}$. six-hourly, brought an initial improvement. One week later she complained of retrosternal pain on swallowing, but otherwise there was no deterioration in her condition, and no cause could be found for her symptoms. Three days later a few patches of thrush appeared in the oropharynx. A barium swallow (Special Plate, Fig. 2 A) showed the thoracic oesophagus to have an irregular outline, but no filling defects could be seen. Examination of the oral lesions revealed monilial spores and pseudomycelia, and the stools now showed yeasts for the first time; subsequent cultures grew C. albicans.

A diagnosis of moniliasis involving the oesophagus was made. Sulphasalazine was discontinued and nystatin was given, as a mucilage, 500,000 units six-hourly. Within 36 hours the dysphagia had lessened and by the fourth day swallowing was normal. The dosage of nystatin was reduced to 250,000 units six-hourly and finally stopped on the ninth day. Subsequent swabs from the

* Registrar in Medicine, St. Bartholomew's Hospital, London.

† Radiologist, St. Bartholomew's Hospital, London. mouth and specimens of stool no longer contained yeasts, and a repeat barium swallow showed the oesophagus to be normal (Special Plate, Fig. 2 B).

\section{Discussion}

Thrush often occurs in patients with blood dyscrasias, and with the exception of Case 2 and two others described by Woods, Manning, and Patterson (1951) and Bernay and Houver (1961), all cases of oesophageal moniliasis have occurred in patients with leukaemia or aplastic anaemia. Superinfection by candida is a well-recognized complication of treatment with antibiotics, either alone, or in combination with corticosteroids. Both the present patients had received combined therapy. The predisposing effect of antibiotics is particularly important, and in only one case of oesophageal moniliasis had they not been given (Bernay and Houver, 1961). On the other hand, several cases have occurred in patients not receiving corticosteroids (Woods et al., 1951 ; Browne, 1954 ; Kaufmann, 1958 ; Eban and Symers, 1959).

In oral thrush, difficulty in swallowing is due solely to local soreness of the mouth, but when infection involves the oesophagus painful retrosternal discomfort develops. This may be very severe (Kaufman, Scheff, and Levene, 1960), and in Case 1 even fluids caused great distress. The pain was not influenced by posture or treatment with antacids, but it is rare for obstructive symptoms to occur (Browne, 1954). When monilial oesophagitis develops secondarily to oral thrush, the cause of the dysphagia is readily apparent, but difficulty arises when it is the predominant manifestation or the presenting symptom of a monilial infection, as was the case in the present two patients. In Case 1 thrush could not be seen at any stage of the disease, while in Case 2 the oropharyngeal lesions were delayed in appearance and never conspicuous. The development of oesophageal moniliasis caused no objective worsening in the general condition of either patient, and a similar lack of constitutional upset was also noted by Eban and Symers (1959) and by Kaufman et al. (1960).

Culture of $C$. albicans from the mouth or stools is of limited value in diagnosis, for it is commonly found in healthy subjects. However, the demonstration of pseudomycelia penetrating mucosal epithelia suggests actual infection. Gastric aspiration is of value in obtaining swallowed pharyngeal and oesophageal secretions for examination when thrush cannot be found in the oropharynx.

The radiological appearances were typical of those previously described (Andrén and Theander, 1956; Kaufman et al., 1960). The outline of the oesophagus becomes shaggy and irregular and the mucosal pattern is lost. As infection proceeds, small circular protrusions and indentations or filling defects due to pseudomembrane appear, and the coating of barium tends to remain adherent to the wall of the oesophagus (Eban and Symers, 1959). The $x$-ray changes are often confined to the thoracic oesophagus, of which the distal third may be the first and, indeed, the only area affected (Kaufmann, 1958 ; Kaufman et al., 1960).

Nystatin is the treatment of choice in monilial oesophagitis and should be given, preferably as a mucilage, 250,000-500,000 units every four to six hours (Marsh, 1959 ; Kaufman et al., 1960). The response to treatment was dramatic in both our 
patients ; improvement in swallowing occurred within 36 hours and complete relief of dysphagia by the fourth day. Rapid healing of the oesophagus occurred, and it was confirmed that the radiological appearances return completely to normal within 10 to 14 days (Kaufman et al., 1960). Amphotericin B is also effective (Egeblad, 1961), but would seem to possess little advantage over nystatin in the treatment of gastro-intestinal moniliasis.

The simultaneous administration of nystatin during broadspectrum antibiotic therapy (Newcomer, Wright, and Sternberg, 1954-5) reduces intestinal overgrowth by candida and should be considered when treating infections in patients who are particularly prone to develop monilial infections.

\section{Summary}

Two cases of painful retrosternal dysphagia due to monilial oesophagitis are described. Oesophageal involvement was the presenting and predominant feature of the monilial infection, there being little evidence of thrush elsewhere. The value of radiology in assisting in diagnosis is emphasized.

Our thanks are due to Dr. E. R. Cullinan and Dr. K. O. Black, under whose care these patients were admitted, for permission to publish details of these cases.

\section{REFERENCES}

Andrén, L., and Theander, G. (1956). Acta radiol. (Stockh.), 46, 571. Bernay, P., and Houver, A. (1961). Arch. Mal. Appar. dig., 50, 233. Browne, S. G. (1954). Lancet, 1, 393.

Eban, R. E., and Symers, D. A. (1959). F. Fac. Radiol. (Lond.), 10, 164. Egeblad, M. (1961). Dan. med. Bull., 8, 163.

Kaufman, S. A., Scheff, S., and Levene, G. (1960). Radiology, 75, 726. Kaufmann, H. J. (1958). New Engl. F. Med., 258, 1143.

Marsh, A. P. (1959). Amer. F. Roentgenol., 82, 1063.

Newcomer, V. D., Wright, E. T., and Sternberg, T. H. (1954-5). Antibiot. Ann., p. 686.

Woods, J. W., Manning, I. H., and Patterson, C. N. (1951). F. Amer. med. Ass., 145, 207.

\section{Medical Memoranda}

\section{Temporary Feeding-gastrostomy- A New Gastrostomy Catheter}

\section{Brit. med. F., 1964, 1, 822}

Gastric decompression with planned replacement of fluid and electrolyte, although of established value in the management of patients having operations on the gastro-intestinal tract, has several disadvantages. Intravenous infusion alarms some patients, and discomfort caused by the naso-gastric tube is complained of by many. The risk of post-operative chest complications may be increased in two ways: the presence of a tube in the nasopharynx discourages coughing; the infusion and aspiration equipment anchor the patient to his bed and limit active movement. Intravenous therapy may cause painful vein-thrombosis. Finally, injury to the larynx and stricture of the lower oesophagus have been attributed to gastric intubation.

It is not surprising, therefore, that some surgeons no longer use gastric aspiration after Polya gastrectomy. However, many consider aspiration advisable after Billroth I gastrectomy and
Studies made in this department during the past two years have provided the following information. Firstly, Ross et al. (1963) have shown that the mean duration of inhibition of small-bowel motility after surgical vagotomy is as little as 10 hours. Secondly, in tests performed at 18 hours after vagotomy, Cox (1962) has shown that there is already a substantial absorption of monosaccharides, electrolytes, and water. These results support the view that post-operative duodenal or jejunal feeding is a satisfactory substitute for intravenous feeding. Finally, in an investigation to be reported, Ross has found complete inhibition of gastric motor activity for at least 36 hours after vagal section, evidence which is in support of the view that gastric aspiration is advisable during the first two days after this operation.

In the light of these findings, a request was made to Messrs. C. F. Thackray Ltd., Park Street, Leeds 1 , to devise a catheter similar to the Foley catheter which would provide a means of gastric aspiration and also permit duodenal feeding after vagotomy with pyloroplasty, or jejunal feeding after vagotomy with gastrojejunostomy. A variety of feeding-gastrostomy tubes have been made for our evaluation. The final product, whose specifications are now given and which has been used by us for six months, is here illustrated.

The three-way F.G. catheter is made of plastic and is fitted with a $20 / 30 \mathrm{ml}$. balloon. Two pairs of opposing eyes on the distal side of the balloon facilitate gastric aspiration. The feed-tube protrudes approximately $11 \mathrm{in} .(28 \mathrm{~cm}$.) beyond the catheter end and has four eyes spirally arranged at the tip. The catheter is supplied sterile.

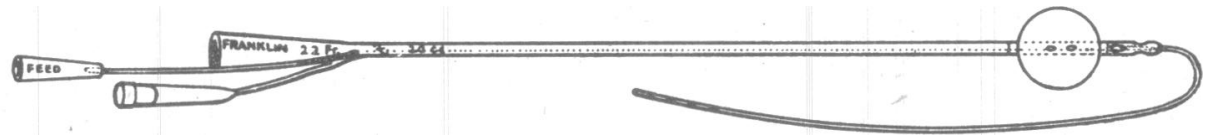

after vagotomy, although it should be noted that Hendry (1962) has claimed excellent results from a regimen of restricted fluids without use of gastric suction.

For almost five years it has been our practice to use a Foley catheter as a temporary gastrostomy in patients who have had vagotomy with a drainage procedure. The catheter is inserted through a small stab incision in the anterior wall of the stomach, drawn through an avascular part of the omentum, and finally withdrawn from the abdominal cavity through a small stab incision in the left epigastrium. This exit wound is usually dry within 24 hours of removing the catheter on the seventh day after operation. Gastric decompression by this means has proved to be a safe procedure and one which obviates the discomforts of a naso-gastric tube. However, fluid and electrolyte replacement may be required in some patients.
Patients unfortunate enough to have had previous experience of gastric surgery have acclaimed this form of post-operative management and recalled the discomfort associated with conventional naso-gastric suction. Intravenous therapy is unnecessary and replacement of electrolytes and fluids via the intestine simplifies the problem of maintaining the composition and distribution of the body fluids. Restriction to bed is unnecessary. A controlled study to determine the incidence of post-operative chest complications in patients with and without nasogastric suction has been planned.

ANDREW W. KAY, Professor of Surgery, Royal Infirmary, Sheffield. REFERENCES

Cox, A. G. (1962). Lancet, 2, 1075.

Hendry, W. G. (1962). Brtt. med. F., 1, 1736.

Ross, B., Watson, B. W., and Kay, A.' W. (1963). Gut, 4, 77. 